\title{
Riesgos psicosociales \\ análisis sociodemográfico y sociolaboral en una universidad privada ecuatoriana
}

Psychosocial risks: sociodemographic and socio-labor analysis in an Ecuadorian private university

(c) (1) $\$(2)$ B E

Ximena Alexandra Ramírez-Ocaña

Marcos Zumárraga-Espinosa Johanna Valeria Segovia-Marín

Edith Evelyn Lima-Acosta Guillermo Marcelo Pillajo-Salcedo 


\section{Psicología \\ ISSN-l: 2027-1786 | e-ISSN: 2500-6517 Publicación Cuatrimestral}

\section{ID: $\quad 10.33881 / 2027-1786 . R I P .13206$}

\section{Title: Psychosocial risks}

Subtitle: Sociodemographic and socio-labor analysis in an ecuadorian private university

Título: Riesgos psicosociales

Subtítulo: Análisis sociodemográfico y sociolaboral en una universidad privada ecuatoriana

Alt Title / Título alternativo:

[en]: Psychosocial risks: Sociodemographic and socio-labor analysis in an Ecuadorian private university

[es]: Riesgos psicosociales: análisis sociodemográfico y sociolaboral en una universidad privada ecuatoriana

Author (s) / Autor (es):

Ramírez-Ocaña, Zumárraga-Espinosa, Segovia-Marín Lima-Acosta \& Pillajo-Salcedo

Keywords / Palabras Clave:

[en]: psychosocial risk factors; sociodemographic and socio-labor analysis; teaching and administrative staff; private university.

[es]: factores de riesgo psicosocial; análisis sociodemográfico y sociolaboral; personal docente y administrativo; universidad privada

Proyecto / Project:

Proyecto de Investigación de Riesgos Psicosociales Laborales

Financiación / Funding:

Universidad Politécnica Salesiana. Quito-Ecuador

Submited: $\quad$ 2020-02-13

Acepted: $\quad 2020-05-21$

\section{Resumen}

Las condiciones actuales de trabajo demandan mayor énfasis en el estudio de los riesgos psicosociales laborales. La presente investigación evalúa dichos riesgos a partir de una muestra conformada por 559 docentes y administrativos de una universidad privada ecuatoriana, con el objetivo de indagar sobre las especificidades de los factores de riesgo

psicosocial en relación con las variables sociodemográficas y sociolaborales, para proponer un perfil de riesgo psicosocial. El instrumento utilizado fue F-PSICO 3.1 y posteriormente a través de regresiones

logísticas se analizó el efecto de dichas variables para identificar posibles factores de riesgo o de protección. Los hallazgos evidencian que la exposición desfavorable a factores de riesgo psicosocial se incrementa en el personal que tiene ciertas características como: ser docente, con jornada de tiempo completo, ser menor de 35 años, contar con menos de 5 años de antigüedad en la institución y ser mujer. A modo de conclusión, al menos el 25\% del personal percibe alguno de los factores psicosociales evaluados de una manera desfavorable.

\section{Abstract}

Current working conditions demand greater emphasis on the study of occupational

psychosocial risks. The present investigation

evaluates these risks from a sample of 559 teachers and administrators of an Ecuadorian private university, with the objective of investigating the specificities of psychosocial risk factors in relation to sociodemographic and socio-labor variables, to propose a profile

of psychosocial risk. The instrument used

was F-PSICO 3.1 and subsequently through logistic regressions the effect of these variables was analyzed to identify possible risk or protection factors. The findings show that unfavorable exposure to psychosocial risk factors is increased in staff that have certain characteristics such as: being a teacher, fulltime, being under 35, having less than 5 years of experience in the institution and to be a woman. In conclusion, at least $25 \%$ of staff perceive some of the psychosocial factors evaluated in an unfavorable way.

\section{Citar como:}

Ramírez-Ocaña, X. A., Zumárraga-Espinosa, M., Segovia-Marín, J. V., Lima-Acosta, E. E. \& Pillajo-Salcedo, G. M. (2020). Riesgos psicosociales: Análisis sociodemográfico y sociolaboral en una universidad privada ecuatoriana. Revista Iberoamericana de Psicología , 13 (2), 57-68. Obtenido de: https:// reviberopsicologia.ibero.edu.co/article/view/1774

Ximena Alexandra Ramírez-Ocaña, MA Psi

Research ID: AAD-9767-2020

ORCID: $\quad$ 0000-0002-2163-1843

Source | Filiacion:

Universidad Politécnica Salesiana (UPS-Ecuador)

BIO:

Docente investigadora de la Universidad Politécnica Salesiana; Miembro del Grupo de Investigaciones Psicosociales (GIPS).

\section{City | Ciudad:}

Quito [ec]

e-mail:

xramirez@ups.edu.ec

Edith Evelyn Lima-Acosta, MA Ing

Research ID: AAO-5289-2020

ORCID: $\quad$ 0000-0002-5519-5226

BIO:

Ingeniera. Electrónica; Especialista en Automatización y Control; Magister en Seguridad y Prevención de Riesgos Laborales Técnico de Seguridad y Salud Ocupacional de la Universidad Politécnica Salesiana.

\section{City | Ciudad:}

Quito [ec]

e-mail:

elima@ups.edu.ec
Marcos Zumárraga-Espinosa, MA Eco

AutorID: $\quad 57200575427$

Research ID: AAE-1487-2020

ORCID: $\quad$ 0000-0001-9930-9005

Source | Filiacion:

Universidad Politécnica Salesiana (UPS-Ecuador)

BIO:

Magíster en Gestión Pública (IAEN - Ecuador); Economista, (UCE). Docente investigador; Miembro del Grupo de Investigaciones Psicosociales (GIPS-UPS) y del Grupo de Innovación Educativa (GIE-UPS)

City | Ciudad:

Quito [ec]

e-mail:

mzumarraga@ups.edu.ec

Dr Guillermo Marcelo Pillajo-Salcedo, MA Psi sp

Research ID: 3382482/quillermo-pillajo/

ORCID: $\quad$ 0000-0002-9018-0409

BIO:

Estudiante de psicología industrial y desarrollo de personas y talento humano, Docente y Consultora en área de riesgos laborales.

City |Ciudad

Quito [ec]

e-mail:

gpillajo@ups.edu.ec

City $\mid$ Ciudad:

Quito [ec]

e-mail:

jsegovia@ups.edu.ec 


\section{Riesgos psicosociales análisis sociodemográfico y sociolaboral en una universidad privada ecuatoriana}

Psychosocial risks: sociodemographic and socio-labor analysis in an Ecuadorian private university

Ximena Alexandra Ramírez-Ocaña

Marcos Zumárraga-Espinosa Johanna Valeria Segovia-Marín

Edith Evelyn Lima-Acosta

Guillermo Marcelo Pillajo-Salcedo

\section{Introducción}

Los cambios económicos y demográficos en las últimas décadas han generado mayores exigencias personales en el ámbito laboral (Sánz, 2011), debido a la alta implicación cognitiva y emocional como respuesta a la competitividad global y organizativa para ofrecer más y mejores servicios con menores costos operacionales (Peiró \& Lira, 2013; Vilaret \& Ortiz, 2013). El ámbito universitario no está exento de esta realidad, dada la variabilidad de actividades que se presentan para el personal docente y administrativo (Botero, 2012; Guamán, Bejarano, \& Gaibor, 2017).

Comprender dichos cambios y la concientización social acerca de sus impactos en la salud han promovido el desarrollo de estudios sobre las condiciones y posibles riesgos psicosociales en el trabajo (Moreno-Jiménez \& Garrosa, 2013). Esos estudios muestran que las exigencias laborales en la actualidad influyen en la salud, tanto en el ámbito físico, como mental y emocional

(Peiró, 2004). Una vez que las demandas laborales, en un entorno cambiante, son cada vez más intensas e implican una amplia gama de alternativas e iniciativas de los empleados (Neffa, 2015), para resolver problemas de diversa índole. 


\section{Riesgos psicosociales}

Análisis sociodemográfico y sociolaboral en una universidad privada ecuatoriana

Según la Organización Internacional del Trabajo (1984), los riesgos psicosociales son "aquellas condiciones presentes en una situación de trabajo, relacionadas con la organización, el contenido y la realización del trabajo susceptibles de afectar tanto al bienestary la salud" (física, psíquica, social) del personal docente y administrativo como al desarrollo del trabajo. No gestionar los factores de riesgo psicosocial de modo oportuno y adecuado, podría incurrir en una experiencia subjetiva negativa hacia el trabajo (Gil-Monte \& Neveu, 2013) y hacia las personas con las que el empleado se relaciona o brinda un servicio, en especial hacia sus clientes (Pinos-Mora, 2017). Durante las últimas décadas las universidades ecuatorianas se han enfrentado a cambios en las políticas públicas y al incremento en la tasa bruta de matrícula, lo que ha significado importantes transformaciones en el ámbito laboral universitario ecuatoriano (Vilaret \& Ortiz, 2013).

Particularmente en las universidades privadas, estos cambios han venido acompañados por una reorganización del trabajo, basada en el nivel de interacción con el estudiantado (Charría, Sarsosa, \& Arenas, 2011). Es decir, emerge una dinámica en la cual el estudiante es el "cliente". De este modo al personal docente se le demanda múltiples actividades a realizar, como la investigación, la innovación, la transferencia de conocimiento (Botero, 2012), la atención a un mayor número de estudiantes (Garrido-Pinzón, Rodríguez-Uribe, \& Blanch, 2011), pero también se le otorgan actividades administrativas. Además, se le exige una constante formación y actualización. Estos factores han influido en la pérdida de la salud generando alteraciones a nivel psicosomático, cambios cognitivos y emocionales (Cladellas \& Castelló, 2011). Por su parte el personal administrativo tiene otras exigencias asociadas a las relaciones con sus compañeros, el tipo de liderazgo de las jefaturas, el trabajo en equipo, la incorrecta gestión del tiempo y una comunicación ineficaz (Gómez-Gómez, López-Cano, \& Munuera-Gómez, 2017). Lo antes expuesto da cuenta de factores causales de posibles riesgos psicosociales (Guamán, Bejarano, \& Gaibor, 2017).

En este sentido, se torna inminente determinar los factores de riesgo psicosocial que operan en los distintos estamentos universitarios: personal docente y administrativo. En este artículo se presentarán los resultados obtenidos en una universidad privada de la ciudad de Quito.

Las investigaciones sobre los riesgos psicosociales en las universidades ecuatorianas son escasas, algunas indagan sobre este asunto en las universidades públicas (Guamán, Bejarano, \& Gaibor, 2017; Gómez-Gómez, López-Cano, \& Munuera-Gómez, 2017). En lo que se refiere a riesgos psicosociales en otros ámbitos, en el país existen más estudios (Salvador, 2018) en el ámbito aeroportuario, (Vega, Vargas, Amores, \& Arias, 2017) en el ámbito textil, (Verdesoto \& Reynaga, 2014) en pequeñas y medianas empresas.

En otros países se ha estudiado este tema con mayor frecuencia. Unda et al. (2016) evaluaron los riesgos psicosociales en $\mathbf{6 2 1}$ docentes universitarios en la Universidad de La Coruña. Terán y Botero (2012), estudiaron los riesgos psicosociales intralaborales en docencia en una muestra de $\mathbf{7 8}$ docentes en Colombia. Y en cuanto al personal administrativo Romero-Díaz, Beleño-Navarro, Ucros-Campo, Echeverría-González, y Lasprilla-Fawcett (2016) estudiaron los factores de riesgo psicosocial en 227 empleados de la Corporación Universidad de la Costa.

Ahora bien, desde el ámbito normativo se evidencia que la prevención del riesgo psicosocial ha sido parte de las políticas públicas. En el año 2013, el (Ministerio de Relaciones Laborales del Ecuador), actualmente (Ministerio del Trabajo), mencionaba que los factores de riesgo psicosocial existen y forman parte de las condiciones de trabajo, y no solo permanecen en la mente del empleado y, por tanto, deben ser evaluados de forma integral. Finalmente, en el año 2017 el MDT planteó, a través del acuerdo ministerial No. 82, erradicar toda forma de discriminación laboral y, además, promover la prevención de riesgos psicosociales, buscando garantizar la aplicación de dicha normativa en empresas del sector público y privado con más de $\mathbf{1 0}$ empleados.

Existen distintos modelos teóricos para explicar los factores de riesgo psicosocial evidenciados en el contexto universitario. Se parte de la noción de que las reacciones frente a una situación psicosocial no es la misma para todos los empleados, sino que dependerá de algunos aspectos personales individuales, condiciones de trabajo, influidos también por el contexto económico, político y social (Pérez \& Nogareda, 2012).

El primer modelo que se propone es el de demandas-control de Karasek (1979) el mismo que analiza la relación entre demandas y recursos. Las demandas son las exigencias físicas y psicológicas que el trabajo implica para la persona, (Arias, Montes, \& Masías, 2014) y se encuentran asociadas con la sobrecarga cuantitativa de trabajo, es decir con la cantidad, volumen, presión de tiempo y niveles de atención e interrupciones que la persona tiene en su lugar de trabajo (Hernández, Salanova, \& Peiró, 2001). Por otro lado, se comprende al control como un recurso asociado a las capacidades de los empleados para una mejor gestión de las tareas; es decir, el control influye sobre la percepción que tiene quien trabaja, acerca de los tipos de demandas a los que se enfrenta, sean estas amenazantes o retadoras (Salanova, 2009).

El segundo modelo de referencia contempla la relación de la salud con la interacción entre demandas y recursos, el modelo de Recursos, Emociones y Demandas laborales (RED) (Salanova, Martínez, \& Llorens, 2014) en este caso, se realiza una distinción entre demandas retadoras, como sobrecarga cuantitativa, refiriéndose a aquellas que pueden generar desarrollo y realización personal; y demandas amenazantes, como el conflicto de rol, como una condición que no contribuye al logro de metas personales (Lepine, Podsakoff, \& Lepine, 2005). El modelo RED, también incluye recursos interpersonales (Salanova, Llorens, Cifre, \& Martínez, 2012), es decir aquellos recursos con los que cuenta el personal y que tienen implicación con algunas características sociodemográficas (Arenas \& Andrade, 2013) como el sexo, la edad, etc. Asimismo, es posible relacionar los aspectos sociolaborales, como la formación académica, el rol, los años de antigüedad en la organización y el tiempo de dedicación, porque podrían ser considerados como factores que potencian o limitan la percepción frente a una actividad laboral (García, Iglesias, Saleta, \& Romay, 2016).

Los anteriores modelos teóricos al relacionarse han constituido la base principal de este estudio, porque se puede observar que las variables de demandas y recursos pueden volverse de riesgo o de protección. De riesgo, porque podrían exponer a los empleados ante situaciones psicosociales vulnerables, donde se disminuirían sus capacidades para enfrentar la cotidianidad (Bravo \& Palucci, 2010) como, por ejemplo, carga de trabajo y demandas psicológicas. Por el contrario, dichas variables podrían convertirse en factores de protección psicosocial, como autonomía, variedad de contenido, interés por el trabajador, relaciones y apoyo social (Madero, Ulibarri, Pereyra, Paredes, \& Briceño, 2013), porque se cuenta con recursos individuales u organizacionales, que contribuyen a reducir las probabilidades de que aparezca un riesgo psicosocial, apoyando al desarrollo del individuo. Estos elementos a corto o mediano plazo servirían como moderadores de los factores de riesgo, dependiendo de la percepción que quien trabaja tenga de las distintas condiciones a las que se enfrente. 
El objetivo de esta investigación es indagar sobre las especificidades de los factores de riesgo psicosocial percibidos en relación con las variables sociodemográficas y sociolaborales del personal docente y administrativo. Finalmente se pretende proponer un perfil de riesgo psicosocial a partir de factores de riesgo y protección.

\section{Método}

\section{Diseño del estudio y participantes}

El estudio tuvo un diseño transversal, no experimental y cuantitativo. Se empleó una muestra no probabilística por conveniencia, con 559 empleados de una universidad privada ecuatoriana, tomándose en cuenta tanto al personal docente $(\mathbf{6 1 . 2 \%})$ como administrativo (38.8\%). El $\mathbf{4 6 . 6 \%}$ de los participantes fueron mujeres y el restante $53.4 \%$ hombres.

\section{Instrumento}

Se aplicó el instrumento F-PSICO 3.1, desarrollado por el Instituto Nacional de Seguridad e Higiene en el Trabajo (INSHT), de España. Este instrumento consta de 86 ítems agrupados en 9 factores de riesgo diferenciados: tiempo de trabajo; autonomía; carga de trabajo; demandas psicológicas; variedad/contenido del trabajo; participación/supervisión; interés por el trabajador/compensación; desempeño de rol; relaciones y apoyo social (Pérez \& Nogareda, 2012).

Cada factor aborda aspectos de la actividad laboral con probabilidad de ejercer efectos negativos sobre la salud de los empleados. Igualmente, estos factores pueden descomponerse en dimensiones según su naturaleza y particularidades. Tal es el caso del factor autonomía, que posee dos componentes: autonomía decisoria y temporal. La carga de trabajo cuenta con tres dimensiones: cantidad y dificultad de la tarea, esfuerzo de atención y presiones de tiempo. Por su parte, las demandas psicológicas se descomponen en exigencias psicológicas y emocionales.

Dado que para la valoración de riesgos el F-PSICO 3.1 asigna pesos específicos para las respuestas de cada ítem, y dicha información tiene un carácter reservado, en el presente trabajo se utilizó una escala tipo Likert convencional con adecuaciones de acuerdo con el contexto de la investigación (P. ej. Siempre o casi siempre $=1 ;$ A menudo = 2; A veces = 3; Nunca o casi nunca = 4). Siguiendo este esquema, se asignaron puntuaciones altas a las alternativas de respuesta que implicaban una percepción más negativa del factor psicosocial correspondiente, mientras que se codificó con puntuaciones bajas a aquellas respuestas que indicaban exposiciones favorables hacia dicho factor. Tal ajuste metodológico resultó necesario para profundizar en la identificación de perfiles de riesgo a partir de características sociodemográficas y sociolaborales.

La fiabilidad de cada escala, según el modelo de valoración implementado, se evaluó por medio del coeficiente Alfa de Cronbach ( $\alpha$ ). Se obtuvieron resultados estadísticamente satisfactorios en todos los casos: Tiempo de trabajo $=0.71$; Autonomía $=0.84$; Carga de trabajo $=0.75$; Demandas psicológicas $=0.80$; Variedad/contenido del trabajo $=0.76$; Supervisión/participación = 0.79; Interés por el trabajador/ compensación = 0.86; Desempeño de rol = 0.90; Relaciones y apoyo social $=0.71$. Los puntajes globales por escala se calcularon sumando los valores registrados en los ítems que las integran.

\section{Procedimiento}

En cuanto al procedimiento efectuado, el instrumento se administró de manera digital a partir de un enlace enviado vía correo electrónico desde la Dirección Técnica de Gestión de Talento Humano al personal docente y administrativo. Además, se incluyó en el primer apartado el consentimiento informado y preguntas formuladas ad-hoc sobre variables sociodemográficas y sociolaborales.

\section{Análisis de datos}

Con el propósito de evaluar empíricamente el objetivo de la investigación se procesaron y analizaron los datos mediante el paquete estadístico SPSS 23. En primera instancia, se emplearon los criterios definidos por el F-PSICO 3.1 para determinar el mayor y menor nivel de riesgo en torno a cada factor psicosocial. Las categorías de riesgo propuestas por este instrumento son: situación adecuada (percentil $<$ P65), riesgo moderado (P65 $\leq$ percentil < P75), riesgo elevado (P75 $\leq$ percentil < P85) y riesgo muy elevado (percentil $\geq$ P85) (Ferrer, Guilera, \& Peró, 2011). Posteriormente se examinó de forma desagregada la relación entre factores psicosociales y un conjunto de variables sociodemográficas y psicosociales, con el fin de identificar posibles factores de riesgo o de protección. Así, mediante regresiones logísticas por pasos sucesivos se exploraron las variables que influyen significativamente en la probabilidad de experimentar una exposición desfavorable frente a cada factor de riesgo psicosocial (categorías de riesgo elevado y muy elevado).

\section{Resultados}

Tomando en cuenta las categorías de riesgo propuestas por el F-PSICO 3.1 se inició con un diagnóstico de las variables sociodemográficas y sociolaborales, además de cada factor psicosocial de interés. Se evidenció que el $\mathbf{3 4 . 3 \%}$ de la muestra se situó en edades de 21 a 35 años; el $\mathbf{4 6 . 2 \%}$ se ubicó en el rango de 36 a 50 años y el restante $\mathbf{1 9 . 5 \%}$ reportó edades superiores a 50 años. El $\mathbf{6 0 . 4 \%}$ del personal tiene estudios de posgrado, el $\mathbf{2 1 . 5 \%}$ estudios de pregrado y el $\mathbf{1 8 . 1 \%}$ registró niveles de instrucción formal inferiores. La antigüedad se clasificó de la siguiente manera: $\mathbf{3 5 . 4 \%}$ con menos de 5 años laborando en la institución, $\mathbf{4 5 . 1 \%}$ reportó una permanencia de 5 a 15 años y el $\mathbf{1 9 . 5 \%}$ tiene más de 15 años de antigüedad. Con respecto al tiempo de dedicación, el $\mathbf{9 1 \%}$ de las personas encuestadas tienen una dedicación de tiempo completo y el restante $\mathbf{9} \%$ de medio tiempo.

Como puede observarse en la figura 1, independientemente del aspecto laboral abordado, al menos el $\mathbf{1 6 \%}$ de los empleados/as reporta percepciones que implican un riesgo muy elevado para la salud en términos psicosociales. Al respecto, dimensiones como variedad/ contenido del trabajo (20.9\%), supervisión/participación (19.9\%) y carga de trabajo (19.5\%) presentan una mayor proporción de empleados/ as en situación de riesgo muy elevado.

Por el contrario, demandas psicológicas $(\mathbf{6 3 . 5 \% )}$, relaciones y apoyo social (61.8\%), al igual que supervisión/participación (61.7\%) cuentan con los porcentajes más altos de personas en situación ade- 


\section{Riesgos psicosociales}

Análisis sociodemográfico y sociolaboral en una universidad privada ecuatoriana

cuada. Además, mientras que el factor tiempo de trabajo tiene a un $\mathbf{1 6 . 1 \%}$ de la planta laboral en situación de riesgo elevado, el factor interés por el trabajador/compensación genera un riesgo moderado para el $\mathbf{1 5 . 1 \%}$ de los empleados. Si bien los niveles de riesgo siguen patro- nes de distribución específicos en función de cada factor psicosocial, una lectura general muestra que en promedio el $\mathbf{1 8} \%$ de los empleados se encuentra en riesgo muy elevado, el $\mathbf{1 1} \%$ en riesgo elevado y el $\mathbf{1 0 . 9} \%$ en riesgo moderado de padecer problemas de salud laboral.

Figura 1. Distribución de participantes por nivel de riesgo según factores psicosociales

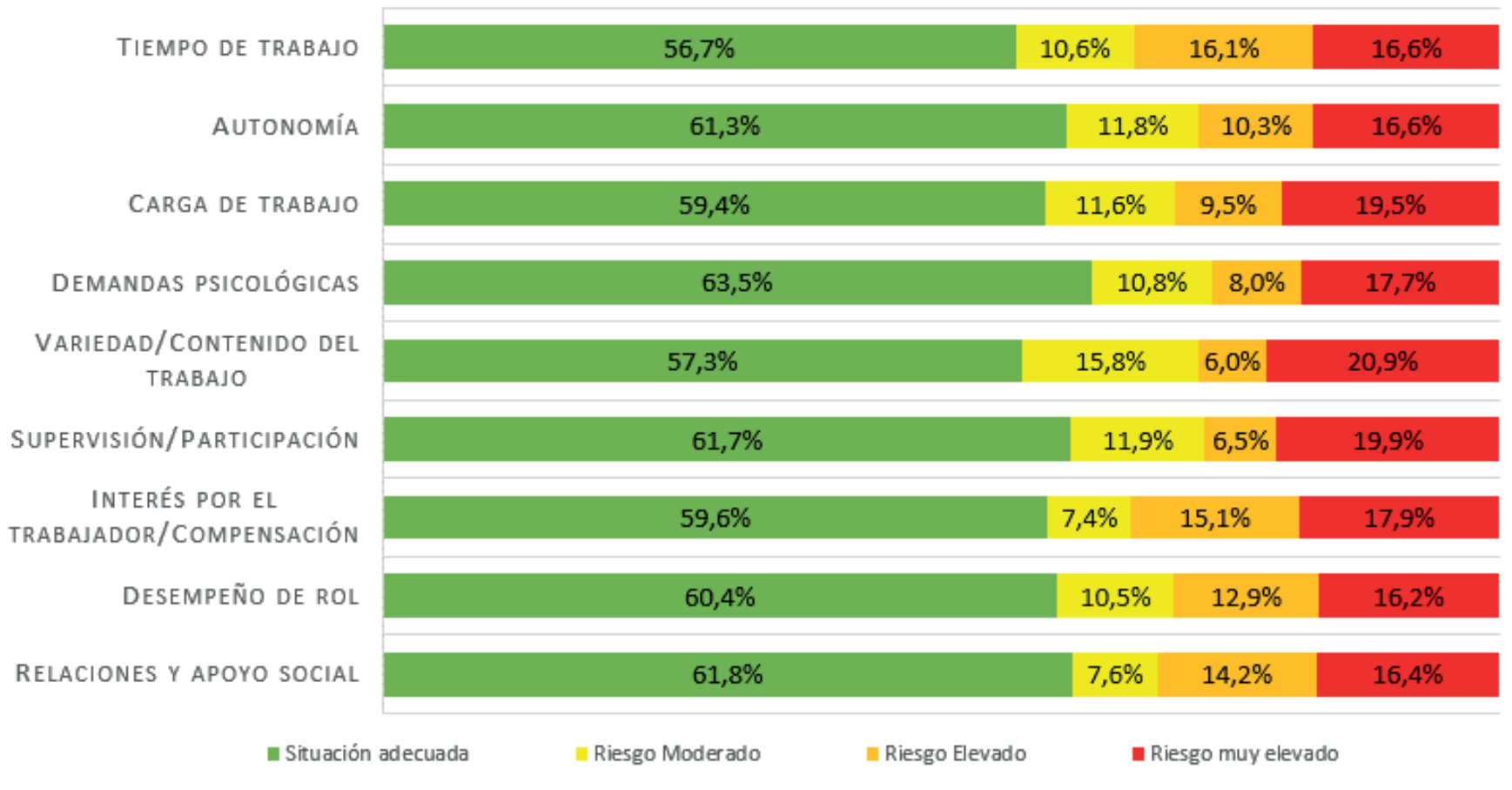

Fuente: datos recolectados por la Dirección Técnica de Gestión de Talento Humano de una universidad privada ecuatoriana, 2019. Elaboración propia.

Puesto que las categorías riesgo elevado y riesgo muy elevado indican una exposición frente a los factores de riesgo psicosocial que resulta potencialmente negativa para la salud, se ha optado por integrar dichas categorías en una sola: exposición psicosocial desfavorable. La tabla 1 muestra, de manera desagregada, el porcentaje de empleados/ as que presenta una exposición desfavorable frente a cada factor de riesgo según distintas características sociodemográficas y sociolaborales.

En términos generales, los resultados muestran que al menos el $\mathbf{2 5 \%}$ de los empleados perciben alguno de los factores psicosociales evaluados de una manera desfavorable. Al desagregar el análisis se observa que en el caso del factor tiempo de trabajo quienes registran mayor prevalencia de exposición a riesgos psicosociales elevados y muy elevados son los empleados con dedicación de medio tiempo (48\%) y el personal docente (44.2\%). Las mujeres (29.8\%), el personal docente (30.4\%) y las personas de 36 a 50 años (29.8\%) experimentan en mayor medida al factor autonomía de modo desfavorable. Lo propio ocurre en los empleados con 35 años o menos (32.8\%) y nuevamente las mujeres (35.3\%) en relación con el factor carga de trabajo.

El factor demandas psicológicas se percibe de manera negativa mayormente por las mujeres (30.6\%), los docentes (30.1\%) y quienes poseen estudios de cuarto nivel (30.4\%). Para el factor variedad/contenido del trabajo son aquellos que no cuentan con formación universitaria (32\%) y tienen de 21 a 35 años (34.4\%) los que enfrentan mayor riesgo psicosocial. En cuanto al factor supervisión/participación, los docentes (30.4\%) y los empleados mayores a 50 años (30.3\%) manifiestan porcentajes más altos de exposición psicosocial desfavorable. El factor interés por el trabajador/compensación se experimenta de manera desfavorable en mayor medida por quienes tienen formación de tercer nivel (39.5\%) y el personal con edades de 21 a 35 años (46.9\%). Situación similar sucede con los empleados que poseen instrucción formal de cuarto nivel (31.3\%) y poseen 35 años o menos (35.4\%) para el factor desempeño de rol. El factor relaciones y apoyo social resulta más riesgoso para quienes tienen de 21 a 35 años (33.9\%), mujeres (33.3\%) y con una antigüedad de 5 a 15 años (33.7\%). 
Tabla 1. Porcentaje de personal docente y administrativo con exposición psicosocial desfavorable por factor y variables personales

\begin{tabular}{|c|c|c|c|c|c|c|c|c|c|}
\hline $\begin{array}{l}\text { Características } \\
\text { sociodemográficas } \\
\text { y sociolaborales }\end{array}$ & $\begin{array}{l}\text { Tiempo de } \\
\text { trabajo }\end{array}$ & Autonomía & $\begin{array}{c}\text { Carga de } \\
\text { trabajo }\end{array}$ & $\begin{array}{c}\text { Demandas } \\
\text { psicológicas }\end{array}$ & $\begin{array}{l}\text { Variedad/ } \\
\text { Contenido del } \\
\text { trabajo }\end{array}$ & $\begin{array}{l}\text { Supervisión/ } \\
\text { Participación }\end{array}$ & $\begin{array}{l}\text { Interés por el } \\
\text { trabajador / } \\
\text { Compensación }\end{array}$ & $\begin{array}{l}\text { Desempeño } \\
\text { de rol }\end{array}$ & $\begin{array}{l}\text { Relaciones y } \\
\text { apoyo social }\end{array}$ \\
\hline \multicolumn{10}{|l|}{ Rol } \\
\hline Administrativo & 14.4 & 22.3 & 27.4 & 19.5 & 30.7 & 20.0 & 38.6 & 26.5 & 30.2 \\
\hline Docente & 44.2 & 30.4 & 30.4 & 30.1 & 24.5 & 30.4 & 29.5 & 31.0 & 31.0 \\
\hline \multicolumn{10}{|l|}{ Sexo } \\
\hline Hombre & 33.4 & 25.0 & 24.0 & 22.0 & 26.4 & 24.0 & 32.1 & 29.4 & 28.4 \\
\hline Mujer & 31.8 & 29.8 & 35.3 & 30.6 & 27.5 & 29.1 & 34.1 & 29.1 & 33.3 \\
\hline \multicolumn{10}{|l|}{ Edad } \\
\hline De 21 a 35 años & 31.3 & 25.0 & 32.8 & 29.2 & 34.4 & 25.5 & 46.9 & 35.4 & 33.9 \\
\hline De 36 a 50 años & 35.6 & 29.8 & 29.5 & 26.0 & 23.6 & 24.8 & 26.0 & 27.1 & 28.3 \\
\hline Más de 50 años & 28.4 & 23.9 & 21.1 & 19.3 & 20.2 & 30.3 & 23.9 & 22.0 & 29.4 \\
\hline \multicolumn{10}{|l|}{ Tiempo de antigüedad } \\
\hline Menos de 5 años & 34.8 & 27.3 & 27.3 & 25.8 & 27.8 & 27.8 & 37.9 & 27.8 & 28.8 \\
\hline De 5 a 15 años & 32.9 & 29.0 & 30.6 & 26.6 & 28.6 & 25.0 & 34.1 & 31.0 & 33.7 \\
\hline Más de 15 años & 27.9 & 22.0 & 28.4 & 23.9 & 20.2 & 25.7 & 20.2 & 26.6 & 25.7 \\
\hline \multicolumn{10}{|l|}{ Formación } \\
\hline Menos de tercer nivel & 17.0 & 24.0 & 25.0 & 15.0 & 32.0 & 29.0 & 30.0 & 25.0 & 33.0 \\
\hline Tercer nivel & 22.7 & 24.4 & 24.4 & 22.7 & 25.2 & 19.3 & 39.5 & 26.9 & 27.7 \\
\hline Cuarto nivel & 40.9 & 29.3 & 32.2 & 30.4 & 26.0 & 28.1 & 31.6 & 31.3 & 31.0 \\
\hline \multicolumn{10}{|l|}{ Tiempo de dedicación } \\
\hline Medio tiempo & 48.0 & 24.0 & 8.0 & 20.0 & 16.0 & 28.0 & 30.0 & 14.0 & 28.0 \\
\hline Tiempo completo & 31.2 & 27.6 & 31.3 & 26.6 & 28.0 & 26.2 & 33.3 & 30.8 & 31.0 \\
\hline
\end{tabular}

Fuente: datos recolectados por la Dirección Técnica de Gestión de Talento Humano de una universidad privada ecuatoriana, 2019. Elaboración propia.

Con el fin de evaluar en qué medida las variables sociodemográficas y sociolaborales se asocian con cada factor de riesgo psicosocial se realizaron análisis de regresión logística binaria por pasos. Para ello se consideró como variable dependiente a la exposición psicosocial desfavorable (riesgo elevado y muy elevado), asignándose el valor de 1 a la presencia de esta condición y 0 a su ausencia. En las tablas 2 y 3 se exponen los resultados de las regresiones logísticas correspondientes a cada factor de riesgo psicosocial, mostrándose únicamente aquellas variables que presentaron relaciones estadísticamente significativas. De existir más de una variable independiente significativa, la estimación de las relaciones mostradas se efectuó controlando la influencia del resto de covariables incluidas en el modelo. Se estimaron coeficientes Odds Ratio (OR) como medida de la fuerza de asociación entre variables (Cerda, Vera, \& Rada, 2013). En el caso de modelos multivariantes se incluyó la prueba de bondad de ajuste de Hosmer y Lemeshow.

El factor tiempo de trabajo (véase tabla 2) se relaciona de manera significativa y negativa con la edad. Observándose que los empleados con edades de 21 a 35 años tienen 2.13 veces $(O R=0.47)$ más riesgo de sufrir una exposición psicosocial desfavorable frente a quienes superan los 50 años ${ }^{1}$. Igualmente, este factor muestra asociación con el rol y

1 Cuando los coeficientes OR son menores a 1 es conveniente invertir su valor y la categoría de referencia (ref.) para facilitar su interpretación. En este sentido, el OR relativo a las probabilidades de sufrir una exposición desfavorable de quienes tienen 50 años o más con respecto a quienes tienen de 21 a 35 años (ref.) es de 0.47, como se reporta en la tabla. Esto indica, en términos generales, que la probabilidad de que los empleados/as con más de 50 años experimenten exposiciones la formación. Es así como los docentes muestran 16 veces más riesgo que las personas que desempeñan cargos administrativos.

Además, los empleados/as con instrucción menor al grado universitario muestran un riesgo 3.85 veces $(O R=0.26)$ más elevado que aquellos con formación de cuarto nivel. En el factor autonomía únicamente se observaron diferencias significativas por rol, siendo los docentes quienes muestran 1.52 veces más probabilidad de experimentar riesgo psicosocial elevado o muy elevado.

El sexo, la edad, la formación y el tiempo de dedicación se relacionaron de modo significativo con la probabilidad de tener una interacción problemática con la dimensión carga de trabajo. Las mujeres tienen 1.85 veces más riesgo que los hombres. La edad parece asociarse negativamente con este factor, los empleados de 21 a 35 años manifiestan 1.85 veces $(O R=0.54)$ más riesgo que aquellos con edades superiores a 50 años. Por el contrario, mayor formación aumenta la probabilidad de padecer problemas de salud laboral. Con referencia a quienes reportan un nivel de educación menor al universitario, los empleados que cuentan con formación de cuarto nivel presentan 1.8 veces más riesgo. Además, trabajar en régimen de tiempo completo produce 5.67 veces más probabilidad de percibir negativamente las condiciones relativas a la carga de trabajo que de existir una dedicación de medio tiempo.

desfavorables es 0.47 veces respecto al caso de quienes tienen 35 años o menos. Dicho de manera inversa, el riesgo de los empleados/as de 21 a 35 años es 2.13 (1/0.47) veces mayores que el de aquellos que superan los 50 años. Se conservará esta segunda forma de interpretación a lo largo de la exposición de los resultados. 


\section{Riesgos psicosociales}

Análisis sociodemográfico y sociolaboral en una universidad privada ecuatoriana

El riesgo psicosocial elevado o muy elevado en el factor demandas psicológicas se conecta con el sexo, la edad y la formación. Tener de 21 a 35 años eleva el riesgo en 1.96 veces $(O R=0.51)$ con respecto a quienes tienen más de 50 años. Asimismo, contar con estudios de cuarto nivel genera 2.94 veces más riesgo que un nivel de formación inferior al tercer nivel. Mientras que las mujeres tienen un riesgo 1.67 veces mayor que los hombres.

El factorvariedad/contenido del trabajo (véase tabla 3) únicamente se relacionó de modo significativo con la edad. Los empleados de 21 a 35 años poseen 1.69 veces $(O R=0.59)$ más riesgo que aquellos con

edades de 36 a 50 años, al igual que $2.08(O R=0.48)$ veces más riesgo que quienes reportaron edades por encima de los 50 años. El factor supervisión/participación se asoció estadísticamente con el rol y la formación. Con respecto a este factor, es 6.08 veces más probable que un docente enfrente niveles peligrosos de riesgo psicosocial en comparación con un empleado administrativo. Entretanto, los empleados con niveles de educación inferiores al grado universitario presentan 2.7 veces $(O R=0.37)$ más riesgo de exposición desfavorable que quienes cuentan con estudios de tercer nivel, así como 5.88 veces $(O R=0.17)$ mayor riesgo que el personal con formación de cuarto nivel.

Tabla 2. Regresiones logísticas multivariantes por factor de riesgo psicosocial: Tiempo de trabajo, Autonomía, Carga de trabajo, Demandas Psicológicas

\begin{tabular}{|c|c|c|c|c|c|}
\hline & $\beta$ & $\begin{array}{l}\text { Error } \\
\text { estándar }\end{array}$ & p & Odds ratio & $\begin{array}{c}\text { Odds ratio } \\
\text { (Intervalo de } \\
\text { confianza al 95\%) }\end{array}$ \\
\hline \multicolumn{6}{|l|}{ FACTOR: Tiempo de trabajo } \\
\hline \multicolumn{6}{|l|}{ Rol } \\
\hline \multicolumn{6}{|l|}{ Administrativo (ref.) } \\
\hline Docente & 2.772 & 0.506 & 0.000 & 16.00 & $5.93-43.15$ \\
\hline \multicolumn{6}{|l|}{ Formación } \\
\hline \multicolumn{6}{|l|}{ Menos de tercer nivel (ref.) } \\
\hline Tercer nivel & -0.354 & 0.395 & 0.370 & 0.70 & $0.32-1.52$ \\
\hline Cuarto nivel & -1.365 & 0.572 & 0.017 & 0.26 & $0.08-0.78$ \\
\hline \multicolumn{6}{|l|}{ Edad } \\
\hline \multicolumn{6}{|l|}{ De 21 a 35 años (ref.) } \\
\hline De 36 a 50 años & -0.256 & 0.229 & 0.263 & 0.77 & $0.49-1.21$ \\
\hline Más de 50 años & -0.750 & 0.290 & 0.010 & 0.47 & $0.27-0.83$ \\
\hline Prueba de Hosmery Lemeshow & \multicolumn{5}{|c|}{$x^{2}=5.722 ; p=0.334$} \\
\hline \multicolumn{6}{|l|}{ FACTOR: Autonomía } \\
\hline \multicolumn{6}{|l|}{ Rol } \\
\hline \multicolumn{6}{|l|}{ Administrativo (ref.) } \\
\hline Docente & 0.418 & 0.202 & 0.039 & 1.52 & $1.02-2.26$ \\
\hline \multicolumn{6}{|l|}{ FACTOR: Carga de trabajo } \\
\hline \multicolumn{6}{|l|}{ Sexo } \\
\hline \multicolumn{6}{|l|}{ Hombre (ref.) } \\
\hline Mujer & 0.615 & 0.197 & 0.002 & 1.85 & $1.26-2.72$ \\
\hline \multicolumn{6}{|l|}{ Edad } \\
\hline \multicolumn{6}{|l|}{ De 21 a 35 años (ref.) } \\
\hline De 36 a 50 años & -0.287 & 0.220 & 0.192 & 0.75 & $0.49-1.15$ \\
\hline Más de 50 años & -0.612 & 0.295 & 0.038 & 0.54 & $0.31-0.97$ \\
\hline \multicolumn{6}{|l|}{ Formación } \\
\hline \multicolumn{6}{|l|}{ Menos de tercer nivel (ref.) } \\
\hline Tercernivel & -0.104 & 0.321 & 0.746 & 0.90 & $0.48-1.69$ \\
\hline Cuarto nivel & 0.590 & 0.269 & 0.028 & 1.80 & $1.06-3.06$ \\
\hline \multicolumn{6}{|l|}{ Tiempode dedicación } \\
\hline \multicolumn{6}{|l|}{ Medio tiempo (ref.) } \\
\hline Tiempo completo & 1.735 & 0.536 & 0.001 & 5.67 & $1.98-16.20$ \\
\hline Prueba de Hosmery Lemeshow & \multicolumn{5}{|c|}{$x^{2}=5.533 ; p=0.595$} \\
\hline \multicolumn{6}{|l|}{ FACTOR: Demandas psicológicas } \\
\hline \multicolumn{6}{|l|}{ Sexo } \\
\hline Hombre (ref.) & & & & & \\
\hline Mujer & 0.509 & 0.203 & 0.012 & 1.66 & $1.12-2.48$ \\
\hline Edad & & & & & \\
\hline De 21 a 35 años (ref.) & & & & & \\
\hline De 36 a 50 años & -0.313 & 0.227 & 0.169 & 0.73 & $0.47-1.14$ \\
\hline Más de 50 años & -0.675 & 0.304 & 0.026 & 0.51 & $0.28-0.92$ \\
\hline Formación & & & & & \\
\hline Menos de tercer nivel (ref.) & & & & & \\
\hline Tercernivel & 0.432 & 0.359 & 0.229 & 1.54 & $0.76-3.11$ \\
\hline Cuarto nivel & 1.077 & 0.312 & 0.001 & 2.94 & $1.59-5.41$ \\
\hline Prueba de Hosmery Lemeshow & & & $=8.771 ; p$ & & \\
\hline
\end{tabular}


Tabla 3. Regresiones logísticas multivariantes por factor de riesgo psicosocial: Variedad/contenido del trabajo, supervisión/participación, Interés por el trabajador/compensación y desempeño de rol

\begin{tabular}{|c|c|c|c|c|c|}
\hline & $\beta$ & $\begin{array}{c}\text { Error } \\
\text { Estándar }\end{array}$ & $\mathrm{p}$ & Odds ratio & $\begin{array}{c}\text { Odds ratio } \\
\text { (Intervalo de } \\
\text { confianza al 95\%) }\end{array}$ \\
\hline \multicolumn{6}{|c|}{ FACTOR: Variedad / Contenido del trabajo } \\
\hline \multicolumn{6}{|l|}{ Edad } \\
\hline \multicolumn{6}{|l|}{ De 21 a 35 años (ref.) } \\
\hline De 36 a 50 años & -0.526 & 0.211 & 0.013 & 0.59 & $0.39-0.89$ \\
\hline Más de 50 años & -0.728 & 0.283 & 0.010 & 0.48 & $0.28-0.84$ \\
\hline \multicolumn{6}{|c|}{ FACTOR: Supervisión / Participación } \\
\hline \multicolumn{6}{|l|}{ Formación } \\
\hline \multicolumn{6}{|l|}{ Menos de tercer nivel (ref.) } \\
\hline Tercer nivel & -0.990 & 0.363 & 0.006 & 0.37 & $0.18-0.76$ \\
\hline Cuarto nivel & -1.794 & 0.525 & 0.001 & 0.17 & $0.06-0.47$ \\
\hline \multicolumn{6}{|l|}{ Rol } \\
\hline \multicolumn{6}{|l|}{ Administrativo (ref.) } \\
\hline Docente & 1.805 & 0.468 & 0.000 & 6.08 & $2.43-15.21$ \\
\hline Prueba de Hosmery Lemeshow & \multicolumn{5}{|c|}{$X^{2}=0.007 ; p=0.996$} \\
\hline \multicolumn{6}{|c|}{ FACTOR: Interés por el trabajador / Compensación } \\
\hline \multicolumn{6}{|l|}{ Edad } \\
\hline \multicolumn{6}{|l|}{ De 21 a 35 años (ref.) } \\
\hline De 36 a 50 años & -0.922 & 0.203 & 0.000 & 0.40 & $0.27-0.59$ \\
\hline Más de 50 años & -1.036 & 0.267 & 0.000 & 0.36 & $0.21-0.60$ \\
\hline \multicolumn{6}{|l|}{ FACTOR: Desempeño de rol } \\
\hline \multicolumn{6}{|l|}{ Edad } \\
\hline \multicolumn{6}{|l|}{ De 21 a 35 años (ref.) } \\
\hline De 36 a 50 años & -0.495 & 0.216 & 0.022 & 0.61 & $0.40-0.93$ \\
\hline Más de 50 años & -0.766 & 0.288 & 0.008 & 0.47 & $0.26-0.82$ \\
\hline \multicolumn{6}{|l|}{ Rol } \\
\hline \multicolumn{6}{|l|}{ Administrativo (ref.) } \\
\hline Docente & 0.444 & 0.207 & 0.032 & 1.56 & $1.04-2.34$ \\
\hline \multicolumn{6}{|l|}{ Tiempo de dedicación } \\
\hline \multicolumn{6}{|l|}{ Medio tiempo (ref.) } \\
\hline Tiempo completo & 1.033 & 0.422 & 0.014 & 2.81 & $1.23-6.43$ \\
\hline Prueba de Hosmer y Lemeshow & & & $=3.829 ;$ & 574 & \\
\hline
\end{tabular}

Fuente: datos recolectados por la Dirección Técnica de Gestión de Talento Humano de una universidad privada ecuatoriana, 2019. Elaboración propia.

La dimensión interés por el trabajador/compensación solo se conectó significativamente con la edad, mostrando un patrón negativo. Quienes tienen edades de 21 a 35 años experimentan 2.5 veces (OR = 0.40) más riesgo que las personas de 36 a 50 años, y $2.78(O R=0.36)$ veces más riesgo que los empleados mayores a 50 años.

Por último, la probabilidad de una exposición riesgosa al factor desempeño de rol se relaciona con la edad, el rol y el tiempo de dedicación. Los empleados con edades de 21 a 35 años cuentan con un riesgo 1.64 veces $(O R=0.61)$ mayor que quienes tienen de 35 a 50 años, simultáneamente este riesgo es 2.13 veces $(\mathrm{OR}=0.47$ ) más alto que aquel experimentado por empleados con edades superiores a los 50 años. El personal docente posee 1.56 veces más riesgo que el personal administrativo. Por último, quienes trabajan con una dedicación de tiempo completo poseen 2.81 veces más riesgo que los empleados a medio tiempo. No se encontraron relaciones significativas en cuanto al factor relaciones y apoyo social. El tiempo de antigüedad en la institución tampoco se asoció significativamente con los factores psicosociales examinados.

Como síntesis de los análisis de regresión logística efectuados, la tabla 4 resume los grupos de empleados que tienen mayor probabilidad de exposición desfavorable con respecto a cada factor de riesgo psicosocial. Aportando, de esta manera, información inicial relevante para la configuración de perfiles de riesgo desagregados, según las especificidades de la variedad de aspectos y condiciones laborales que conforman el ámbito universitario. 


\section{Riesgos psicosociales}

Análisis sociodemográfico y sociolaboral en una universidad privada ecuatoriana

Tabla 4. Perfiles de riesgo por factor psicosocial

\begin{tabular}{|c|c|}
\hline \begin{tabular}{|c|}
\hline Factor de riesgo psicosocial \\
\hline Tiempo de trabajo
\end{tabular} & \begin{tabular}{c} 
Perfil de riesgo (Grupos con mayor probabilidad de exposición desfavorable) \\
\hline Autonomía
\end{tabular} \\
\hline Carga de trabajo & Personal docente; formación inferior a tercer nivel; edades de 21 a 35 años \\
\hline Demandas psicológicas & Mujeres; formación de cuarto nivel; edades de 21 a 35 años; dedicación a tiempo completo \\
\hline Variedad/Contenido del trabajo & Mujeres; formación de cuarto nivel; edades de 21 a 35 años \\
\hline Supervisión/Participación & Edades de 21 a 35 años \\
\hline Interés por el trabajador/Compensación & Personal docente; formación inferior a tercer nivel \\
\hline Desempeño de rol & Edades de 21 a 35 años \\
\hline
\end{tabular}

De forma general, los resultados sugieren que características como ser hombre y tener más edad se inclinan a operar como factores protectores. Es así como los empleados de mayor edad se encuentran menos propensos a situaciones de riesgo psicosocial elevado o muy elevado, especialmente si sobrepasan los 50 años. De igual manera, la probabilidad de exposición psicosocial desfavorable tiende a reducirse en los hombres. En cuanto al tiempo de dedicación, trabajar en régimen de tiempo completo constituye un factor de riesgo. De la misma manera, el rol docente tiende a mostrar mayores probabilidades de exposición psicosocial riesgosa, constituyendo otro factor de riesgo. En cambio, la formación proporciona resultados mixtos, pues, aunque actúa como un factor de riesgo en dimensiones psicosociales como demandas psicológicas y carga de trabajo, también opera como factor protector para la dimensión supervisión/participación y tiempo de trabajo. En otras palabras, si bien quienes poseen formación de cuarto nivel experimentan mayores riesgos para la salud laboral en términos de demandas psicológicas y carga de trabajo, sus riesgos psicosociales disminuyen en aspectos como el tiempo de trabajo y la supervisión/ participación.

\section{Discusión y conclusiones}

En la investigación se exploraron las variables sociodemográficas y sociolaborales que pueden modificar la probabilidad de una exposición desfavorable frente a cada factor psicosocial, buscando potenciales factores de riesgo y de protección. A partir de las regresiones logísticas desarrolladas se detectaron ciertos patrones que, aunque no son recurrentes y significativos para la totalidad de factores considerados por el F-PSICO 3.1, aportan indicios para la construcción inicial de un perfil de riesgo con miras a una posterior verificación mediante estudios de mayor profundidad.

Los resultados evidencian el aumento en el nivel de riesgo psicosocial para las mujeres, docentes y personal de tiempo completo. Esto coincide con la investigación realizada por García, Iglesias, Saleta, y Romay (2016), en donde se sugiere que el desequilibrio entre responsabilidades familiares y domésticas es una de las potenciales causas del estrés en las mujeres docentes (doble presencia). De igual manera, el estudio realizado por Barbosa, Muñoz, Rueda, y Suárez (2009) encuentra que las mujeres a diferencia que los hombres utilizan más estrategias de afrontamiento frente al síndrome de burnout, como un riesgo psicosocial. Por otro lado, los resultados obtenidos discrepan con el estudio realizado por Gómez-Gómez, López-Cano, y Munuera-Gómez
(2017) , en una universidad ecuatoriana, mismo que revela que las muejes experiementan menores niveles de riesgo psicosocial que los hombres, sean estos docentes o administrativos, mencionando que es posible que se deba a que los hombres pueden no ver en la mujer a una posible competidora o no confían en su trabajo, lo que podría ser un factor condicionate en el ámbito laboral (De Cabo, Henar, \& Calvo, 2009).

En relación con la edad, el riesgo aumenta para los empleados de 21 a 35 años. Al respecto el análisis realizado por Pujol-Cols (2017) coincide al señalar que las personas mayores de 40 años perciben una exposición menor a factores de riesgo como exigencias psicológicas y percepción de insuficiente autonomía, comparado con empleados menores de 40 años. Estos resultados difieren de los reportes realizados en el estudio de Domínguez-Gómez (2004), en donde se observa un nivel de estrés elevado en los profesores entre 46 y 50 años.

Diversos autores mantienen que entre algunos factores que se relacionan con la satisfacción laboral, se encuentran el volumen de horas de trabajo, los plazos, los horarios que regulan la actividad laboral y otros factores que influyen sobre la gestión del tiempo.

Es así como, en el presente estudio se pueden observar niveles elevados de riesgo psicosocial en empleados con un contrato a tiempo completo, a diferencia de aquellos con contrato a tiempo parcial. Pese a que en los estudios realizados surgen resultados similares, se destaca que ninguno de estos dos grupos se encuentra estresado (DomínguezGómez, 2004; Cladellas \& Badía-Martín, 2010). En cuanto a la formación académica, la presente investigación la describe como un patrón más heterogéneo, desempeñándose tanto como factor de riesgo y factor protector dependiendo de la dimensión laboral que se analice.

Respecto a los factores que inciden directamente en un perfil de riesgo psicosocial, se encuentran aquellos relacionados con el rol que desempeña el empleado en la institución académica, en este caso, el personal docente constituye el perfil con mayor riesgo a exposición a riesgos psicosociales. Existe una diversidad de estudios sobre las condiciones laborales del docente y sus efectos sobre la salud, que denotan la relación entre la docencia y los factores de riesgo psicosocial (Cladellas \& Castelló, 2011) . En más de uno de éstos se demuestra que el rol docente enfrenta demandas laborales de diversa índole (Garrido-Pinzón, Rodríguez-Uribe, \& Blanch, 2011), la forma en que está estructurado su trabajo en la actualidad y las diversas condiciones a las que se encuentra expuesto como evaluaciones, formatos, compra de libros, material, etc., (Unda, y otros, 2016), son actividades que exigen un mayor esfuerzo cualitativo y cuantitativo en el docente, lo que podría ocasionar un mayor desgaste en su salud (Terán \& Botero, 2012). 
Otro factor con relación directa es la antigüedad en el puesto de trabajo. La presente investigación revela que el nivel de riesgo psicosocial se incrementa en los empleados con una antigüedad menor a 5 años. Resultados similares se pueden apreciar en estudios en los que los docentes con menor experiencia en sus funciones experimentan niveles superiores de estrés a diferencia de docentes con más de 10 años en la profesión (Viloria \& Paredes, 2002). Datos corroborativos se encuentran en estudios realizados en docentes universitarios, aquí la relación entre antigüedad y estrés es directa, siendo los empleados con menos de 5 años y aquellos entre 11 y 15 años de antigüedad en sus funciones, los grupos más expuestos al estrés (Domínguez-Gómez, 2004).

En consecuencia, un primer acercamiento a un perfil de riesgo para los empleados en el entorno universitario; situaría a las mujeres, docentes, de menos edad, con menor antigüedad (Madero, Ulibarri, Pereyra, Paredes, \& Briceño, 2013) en la institución y que trabajen bajo modalidad de tiempo completo, como el grupo más vulnerable ante riesgos psicosociales y sus repercusiones en la salud.

Este trabajo analiza el comportamiento de diferentes factores de riesgo psicosocial en el entorno universitario, en relación con variables sociodemográficas y sociolaborales en personal docente y administrativo. Los resultados indican que, en promedio, cerca de la tercera parte de los empleados perciben los factores psicosociales evaluados de una manera desfavorable, implicando riesgos elevados y muy elevados, de padecer problemas de salud física, psicológica y social.

Considerando la base teórica que resalta a los recursos de la persona como un factor determinante para enfrentar las demandas del entorno, al igual que la influencia de las capacidades de los empleados sobre la percepción y respuesta hacia las demandas laborales; se puede confirmar que cuando existen demandas laborales que sobrepasan el control o los recursos (sean sociodemográficos o sociolaborales) con los que cuentan los empleados, existe una probabilidad más alta de que se presenten riesgos psicosociales.

Los factores de riesgo psicosocial asociados con la variedad de contenido del trabajo, supervisión/participación y carga de trabajo, muestran una mayor incidencia (riesgo muy elevado). Se observa también, que los factores asociados a demandas psicológicas relaciones y apoyo social se encuentran en una situación adecuada. A pesar de que el factor supervisión/participación, se encuentra en un nivel de riesgo muy elevado (19.5\%), también se observa que un alto porcentaje se encuentra en situación adecuada $(\mathbf{6 1}, \mathbf{7 \%})$.

Entre las variables que evidencian una mayor relación con factores de riesgo psicosocial se encuentran el sexo, la edad, el tiempo de trabajo y el rol que desempeña el empleado en la universidad, en este caso personal docente. Es posible interpretar que este perfil se encuentra expuesto a mayor riesgo psicosocial, debido a la naturaleza sus responsabilidades, las circunstancias en las que se encuentra, así como el entorno cambiante y altamente demandante en el que se desempeña.

Con los aspectos mencionados, será importante reflexionar sobre la importancia de generar propuestas, a fin de ejercer una influencia positiva en la percepción de los empleados hacia los factores de riesgo psicosocial, con énfasis en factores como variedad y contenido del trabajo, carga de trabajo o supervisión/participación. Respecto a variables tales como rol, sexo, edad y tiempo de trabajo, se requieren estrategias focalizadas en función de los perfiles de riesgo identificados, con el objetivo de mejorar la forma en que se enfrentan los riesgos psicosociales y fomentar la salud psicosocial en el trabajo.
En cuanto a las limitaciones del estudio, puesto que se efectuaron ajustes metodológicos para la aplicación del F-PSICO 3.1, los resultados aquí expuestos requieren de esfuerzos adicionales de verificación, tanto a partir de las escalas de valoración originales del instrumento, como de otras escalas que aborden los factores de riesgo psicosociales analizados. De igual manera, una vez examinado el efecto de variables sociodemográficas y académicas, resulta necesario avanzar hacia la detección de otros factores, con mayor poder explicativo, que se relacionen con las diferentes formas de riesgo psicosocial que pueden tener lugar en el ambiente universitario. Esto con el fin de robustecer la comprensión y construcción de perfiles de riesgo, de cara al desarrollo de intervenciones más efectivas en favor de la salud mental del personal administrativo y docente.

\section{Referencias}

Arenas, F., \& Andrade, V. (2013). Factores de riesgo psicosocial en una industria alimenticia de la ciudad de Cali. Pensamiento Psicológico, 11(1), 99-113. Obtenido de https://revistas.javerianacali.edu.co/index. php/pensamientopsicologico/article/view/420

Arias, W., Montes, I., \& Masías, M. (2014). El modelo de demanda control de Karasek y su relación con la creatividad docente en profesores de nivel primario de Arequipa. Revista de Psicología (Trujillo), 16(1), 64-77. Obtenido de http://181.224.246.204/index.php/R_PSI/article/ view/236/139

Barbosa, L., Muñoz, M., Rueda, P., \& Suárez, K. (2009). Síndrome de burnout y estrategias de afrontamiento en docente universitarios. Revista Iberoamericana de Psicología: Ciencia y Tecnología, 2(1), 21-30. Obtenido de https://reviberopsicologia.ibero.edu.co/article/ view/174/597

Botero, C. (2012). Work-related psychosocial risk and "burnout" in academic personnel of some latin american countries. Cuadernos de administración, 28(48), 117-132. Obtenido de http://www.scielo.org. co/pdf/cuadm/v28n48/v28n48a10.pdf

Bravo, C., \& Palucci, M. (2010). El consumo de alcohol en personal administrativo y de servicios de una universidad del Ecuador. Revista Latinoamericana Enfermagem, 18(1), 487-495. Obtenido de https:// www.redalyc.org/pdf/2814/281421939002.pdf

Cerda, J., Vera, C., \& Rada, G. (2013). Odds ratio: aspectos teóricos y prácticos. Revista médica de Chile, 141(10), 1329-1335. doi:http:// dx.doi.org/10.4067/S0034-98872013001000014

Charría, V., Sarsosa, K., \& Arenas, F. (2011). Factores de riesgo psicosocial laboral: métodos e instrumentos de evaluación. Revista Facultad Nacional de Salud Pública, 29(4), 380-391. Obtenido de https://wWw. redalyc.org/articulo.oa?id $=12021522004$

Cladellas, R., \& Badía-Martín, M. (2010). La gestion del tiempo de los profesores universitarios en función de la modalidad educativa: sus efectos psicosociales. Revista Española de Pedagogía, 68(246), 297-310. Obtenido de https://dialnet.unirioja.es/servlet/ articulo? codigo $=3206697$

Cladellas, R., \& Castelló, A. (2011). University Professors' Stress and perceived state of health in relation to teaching schedules. Electronic Journal of Research in Educational Psychology, 9(1), 217-240. Obtenido de https://core.ac.uk/download/pdf/143458632.pdf

De Cabo, G., Henar, L., \& Calvo, M. (9 de Junio de 2009). Análisis de la perspectiva de género en algunas estadísticas españolas y propuestas de mejora. Obtenido de Instituto de la Mujer (Ministerio de Igualdad): http://www.inmujer.gob.es/observatorios/ observlgualdad/estudios/nformes/docs/016-analisis.pdf

Domínguez-Gómez, J. (2004). Riesgo psicosocial en la universidad: estresores propios del docente universitario. Revista digital de prevención, 2(2), 1-38. Obtenido de http://rabida.uhu.es/dspace/ handle/10272/4845 


\section{Riesgos psicosociales}

Análisis sociodemográfico y sociolaboral en una universidad privada ecuatoriana

Ferrer, R., Guilera, G., \& Peró, M. (Abril de 2011). Instituto Nacional de Seguridad e Higiene en el Trabajo. Obtenido de https://www. insst.es/InshtWeb/Contenidos/Documentacion/NOVEDADES\%20 EDITORIALES/FPsico/Informe\%20justificaci\%c3\%b3n.pdf

García, M., Iglesias, S., Saleta, M., \& Romay, J. (2016). Riesgos psicosociales en el profesorado de enseñanza universitaria: diagnóstico y prevención. Journal of Work and Organizational Psychology, 32(1), 173-182. doi:http://dx.doi.org/10.1016/j.rpto.2016.07.001.

Garrido-Pinzón, J., Rodríguez-Uribe, A., \& Blanch, J. (2011). Riesgos psicosociales desde la perspectiva de la calidad de vida laboral. Acta colombiana de psicología, 14(2), 27-34. Obtenido de http://WWW. scielo.org.co/pdf/acp/v14n2/v14n2a03.pdf

Gil-Monte, P., \& Neveu, J. P. (2013). El síndrome de quemarse por el trabajo. Madrid, España: Ediciones Pirámide.

Gómez-Gómez, F., López-Cano, A., \& Munuera-Gómez, M. (2017). Riesgos psicosociales en una Universidad de Ecuador: Mediante técnicas de Lógica Difusa. Revista Lusófona de Educação, 34(1), 63-81. Obtenido de http://recil.grupolusofona.pt/handle/10437/7860

Guamán, M., Bejarano, B., \& Gaibor, I. (2017). Los riesgos psicosociales y la satisfacción laboral: Un estudio descriptivo de corte transeccional aplicado al personal administrativo de instituciones de Educación Superior en la República del Ecuador. Publicando, 4(12), 711-729. Obtenido de https://revistapublicando.org/revista/index.php/crv/ article/view/754/pdf 545

Hernández, P., Salanova, M., \& Peiró, J. (2001). El estrés laboral: ¿un concepto cajón de sastre? Proyecto social. Revista de relaciones laborales, 10(11), 167-185. Obtenido de https://dialnet.unirioja.es/ servlet/articulo? codigo $=793102$

Karasek, R. (1979). Job Demands, Job Decision Latitude, and Mental Strain: Implications for Job Redesign. Sage Publications, Inc, 24(2), 285-308. doi:10.2307/2392498

Lepine, J., Podsakoff, N., \& Lepine, M. (2005). A meta-analityc test of the challenge stressor-hindrance stressor framework: an explanation forn inconsistent relationships among stressors and performance. Academy of Management Journal, 48(5), 764-775. doi:https://www. jstor.org/stable/20159696

Madero, J., Ulibarri, H., Pereyra, A., Paredes, F., \& Briceño, M. (2013). Burnout en personal administrativo de una institución de educación superior tecnológica de Yucatán, México. Revista electrónica de psicología Iztacala, 16(3), 741-763. Obtenido de https://www.medigraphic.com/ cgi-bin/new/resumen.cgi?IDARTICULO=45627

Ministerio de Relaciones Laborales del Ecuador. (21 de Septiembre de 2013). Ministerio de Relaciones Laborales del Ecuador. Obtenido de http://www.trabajo.gob.ec/wp-content/uploads/2012/10/NT-25Factores-y-Riesgos-Psicosociales.pdf

Ministerio del Trabajo. (Octubre de 2018). Ministerio del Trabajo del Ecuador. Recuperado el 9 de Junio de 2019, de http://sut.trabajo.gob. ec/mrl/publico/archivoFrm.xhtml\#no-back-button

Moreno-Jiménez, B., \& Garrosa, E. (2013). Salud laboral. Riesgos laborales psicosociales y bienestar laboral. Madrid: Ediciones Pirámide.

Neffa, J. (2015). Los riesgos psicosociales en el trabajo. Una contribución al estudio. Buenos Aires: Centro de estudios e investigaciones laborales- CEIL-CONICET. Obtenido de https://core.ac.uk/ reader $/ 35154340$

Organización Internacional del Trabajo. (1984). Comité Mixto OITOMS sobre Medicina del Trabajo. Obtenido de http://WwW. factorespsicosociales.com/wp-content/uploads/2019/02/FPS-OIT-OMS. $p d f$

Peiró, J. M. (2004). El sistema de trabajo y sus implicaciones para la prevención de los riesgos psicosociales en el trabajo. Universitas psychologica, 3(2), 179-186. Obtenido de http://www.integraorg.com/ wp-content/docs/El\%20sistema\%20de\%20trabajo\%20y\%20sus\%20 implicaciones.pdf
Peiró., J., \& Lira., E. (2013). Estrés laboral: nuevas y viejas fórmulas. En B. Moreno-Jiménez., \& E. Garrosa, Salud laboral. Riesgos laborales psicosociales y bienestar laboral (págs. 103-118). Madrid: Ediciones Pirámide.

Pérez, J., \& Nogareda, C. (2012). Instituto Nacional de Seguridad e Higiene en el Trabajo. Obtenido de Notas técnicas de prevención: https://www.insst.es/InshtWeb/Contenidos/Documentacion/NTP/ NTP/926a937/926w.pdf

Pinos-Mora, L. (2017). Factores psicosociales en la gestión de riesgo laboral. Ciencia de Seguridad y Defensa, 2(1), 79-104. Obtenido de http://geo1.espe.edu.ec/wp-content/uploads/2017/10/art7.pdf

Pujol-Cols, L. (2017). Riesgos psicosociales y satisfacción laboral en docentes de una universidad pública argentina: Explorando el rol del work engagement. Buenos Aires: DEAS Working Paper Series from RePEc. Obtenido de Universidad Nacional de Mar del Plata, Facultad de Ciencias Económicas y Sociales, Centro de Documentación, Nülan. Deposited Documents: http://nulan.mdp.edu. ar/2867/1/pujol-2017.pdf

Romero, C., Beleño, R., Ucros, M., Echeverría, A., \& Lasprilla, S. (2016). Factores de riesgos psicosociales extralaborales en personal administrativo universitario. Revista electrónica enfermería actual en Costa Rica(31), 1-15. doi:10.15517/revenf.v0i31.22083.

Salanova, M. (2009). Psicología de la salud ocupacional (Primera ed.). Madrid: Síntesis. Obtenido de http://www.want.uji.es/wp-content/ uploads/2017/10/2010 Salanova-Intro-Psicolog\%C3\%ADa-de-laSalud-Ocupacional.pdf

Salanova, M., Llorens, S., Cifre, E., \& Martínez, I. (2012). We Need a Hero! Toward a Validation of the Healthy and Resilient Organization (HERO) model. Group \& Organization Management, 37(6), 785-822. doi:10.1177/1059601112470405

Salanova, M., Martínez, I., \& Llorens, S. (2014). Una mirada más "positiva" a la salud ocupacional desde la psicología organizacional positiva en tiempos de crisis: aportaciones desde el equipo de investigación WoNT. Papeles del Psicólogo, 35(1), 22-30. Obtenido de http://WwW. papelesdelpsicologo.es/pdf/2318.pdf

Salvador, J. (2018). Riesgos psicosociales del sector aeroportuario de Manta. Revista San Gregorio, 1(22), 30-39. doi:10.36097/rsan.v1i22.610

Sánz, A. (2011). Work-family balance and occupational health: Is this relationship possible? Current situation in the study of work-family conflict and recovery from stress. Medicine and safety at work, 1(57), 115-126. doi:10.4321/S0465-546X2011000500008.

Terán, A., \& Botero, C. (2012). Riesgos psicosociales intralaborales en docencia. Revista Iberoamericana de Psicología: Ciencia y Tecnología, 5(2), 95-106. doi:10.33881/2027-1786.rip.5210

Unda, S., Uribe, F., Jurado, S., García, M., Tovalín, H., \& Juárez, A. (2016). Elaboración de una escala para valorar los factores de riesgo psicosocial en el trabajo de profesores universitarios. Journal of Work and Organizational Psychology, 32(2), 67-74. doi:10.1016/j. rpto.2016.04.004

Vega, J., Vargas, M., Amores, P., \& Arias, S. (2017). Riesgos psicosociales y la seguridad industrial en las lavanderías textiles del Cantón Pelileo. Revista de Comunicación de la SEECI(43), 135-149. doi:10.15198/ seeci.2017.43.135-149

Verdesoto, Á., \& Reynaga, P. (2014). Factores de riesgos psicosociales y calidad de vida en trabajadores de pequeñas y medianas empresas de Quito-Ecuador. Revista Mexicana de Salud en el Trabajo, 6(16), 48-55. Obtenido de http://fenastac.org.mx/wp-content/_ uploads/2015/02/REMESAT-EDIC1\%C3\%93N-16.pdf\#page $=5$

Vilaret, A., \& Ortiz, Á. (2013). Carga mental y Síndrome de Burnout en docentes a tiempo completo de una universidad de Quito. Eídos, 1(6), 36-47. doi:10.29019/eidos.v0i6.106

Viloria, H., \& Paredes, M. (2002). Estudio del síndrome de Burnout o desgaste profesional en los profesores de la Universidad de Los Andes. Educere, 6(17), 29-36. Obtenido de https://www.redalyc.org/ articulo.oa?id $=356 / 35601704$ 